\title{
Crossover behavior in a mixed-mode fiber bundle model
}

\author{
Srutarshi Pradhan ${ }^{a}$ * Bikas K. Chakrabarti ${ }^{b}$ i and Alex Hansen 0 团 \\ ${ }^{a}$ Department of Physics, Norwegian University of Science and Technology, Trondheim 7491, Norway and \\ ${ }^{b}$ Condensed Matter Physics Group, Saha Institute of Nuclear Physics, \\ 1/AF , Bidhan Nagar, Kolkata -700 064, India
}

\begin{abstract}
We introduce a mixed-mode load sharing scheme in fiber bundle model. This model reduces exactly to equal load sharing (ELS) and local load sharing (LLS) models at the two extreme limits of a single load sharing parameter. We identify two distinct regimes: a) Mean-field regime where ELS mode dominates and b) short range regime dominated by LLS mode. The crossover behavior is explored through the numerical study of strength variation, the avalanche statistics, susceptibility and relaxation time variations, the correlations among the broken fibers and their cluster analysis. Analyzing the moments of the cluster size distributions we locate the crossover point of these regimes. We thus conclude that even in one dimension, fiber bundle model shows crossover behavior from mean-field to short range interactions.
\end{abstract}

\section{INTRODUCTION}

Fiber bundle model represents a simple, stochastic fracture-failure process [1] in materials subjected to external load. The model consists of three basic ingredients: (a) a discrete set of $N$ elements located at sites of a lattice (b) a probability distribution of the strength threshold of individual elements (fibers) (c) a load-transfer rule which distributes the terminal load carried by the failed fibers to the surviving fibers. The model study was initiated by Peirce [2] in the context of testing the strength of cotton yarns. Since then this model has been studied and modified by many groups [3-25] using analytic as well as numerical methods. Fiber bundles are of two classes with respect to the time dependence of fiber strength threshold: 'Static' bundles contain fibers whose threshold strengths are independent of time and such bundles are subjected to quasi static loading, i.e., the load is increased steadily up to the complete failure of the bundles. The load or stress $\sigma$ (load per fiber) is an independent variable here and the strength of the bundle is determined by the maximum value of the applied load or stress $\left(\sigma_{c}\right)$ that can be supported by the bundle. On the other hand 'dynamic' bundles con-

*Electronic address: pradhan.srutarshi@phys.ntnu.no

$\dagger$ Electronic address: bikas@cmp.saha.ernet.in

‡Electronic address: alex.hansen@phys.ntnu.no sist of fibers having time dependent strength and the fibers fail due to fatigue [4, $5,6,6]$ after a period of time which varies fiber to fiber. The time taken for complete failure is called the lifetime of the bundle. According to the load sharing rule, fiber bundles are being classified into two groups: Equal loadsharing (ELS) bundles [8-17] or democratic bundles and local load-sharing (LLS) bundles 18, 19, 20]. In the ELS models all the intact fibers equally share the terminal load of a failed fiber, whereas in LLS model the terminal load gets shared among the intact nearest neighbors. ELS models show phase transition from partial failure to total failure at a critical strength $\left(\sigma_{c}\right)$. The critical behavior in the failure dynamics of ELS bundles has been solved analytically 15, 16 and the universality of the ELS model has been established [17] recently. However the strength of LLS models goes to zero [21, 22, 23] at the limit of infinite system size and this does not permit any critical behavior in the failure process.

The ELS and LLS models belong to two opposite extremes with respect to the spatial correlations in stress redistributions. These models do not incorporate any type of stress gradient among the intact fibers which is an usual expectation. Therefore a load sharing scheme in between ELS and LLS should be a realistic approach to study the failure of heterogeneous materials. Hansen and Hemmer 24] introduced a ' $\lambda$ model' to interpolate between ELS and LLS models where $\lambda$ is an adjustable stress transfer factor. Although they 
conjectured the existence of a critical crossover value $\lambda_{c}$ which separates the mean field (ELS) regime and the short range (LLS) regime, what would be the exact crossover point was not answered. A recent approach by Hidalgo et al [25] incorporate both the ELS and LLS mode introducing an effective range of interaction parameter $(\gamma)$ which is actually the power of the stress redistribution function. They observed crossover behavior in strength variation and in the avalanche statistics of the failures. Also they determined the crossover point $\left(\gamma_{c}\right)$ through the moment analysis of the cluster size distributions before total failure.

In this paper we develop a mixed-mode load sharing (MMLS) model which interpolates the ELS and LLS models correctly. We intend to study whether this model shows a continuous transition from mean-field (ELS) behavior to extreme statistics (LLS), or there exists a definite crossover point.

We organize this paper as follows: After introduction (section I) we present our MMLS model in section II. Section III contains the observations of Crossover behavior through numerical study of the model. The analysis to determine exact crossover point is given in section IV. The final section (V) is devoted for discussions including our conclusions.

\section{THE MODEL}

Our mixed-mode load sharing (MMLS) scheme is basically a coupling of ELS and LLS mode: When a fiber fails, a fraction $(g)$ of its terminal load gets shared among the nearest neighbors of the failed fiber (LLS rule) and the rest $(1-g$ fraction) is distributed equally among all the surviving fibers (ELS rule). Here ' $g$ ' is the weight parameter of the MMLS scheme. Therefore, the model reduces exactly to ELS model for $g=0$ and for $g=1$, it becomes pure LLS one. As we have chosen $1-D$ fiber bundle model (with periodic boundary condition), the number of nearest neighbors is always two. We study the behavior of the model for the entire range $0 \leq g \leq 1$ using Monte-Carlo simulations for step-wise equal load increment 15, 16, 17] until the total failure of the bundle. During the entire study we consider uniform (on average) distribution of fiber strength threshold in the bundle.

\section{THE CROSSOVER BEHAVIOR}

\section{A. Strength of the bundle}

It is known since Daniels 3] that the ELS bundles have a nonzero strength $\left(\sigma_{c}\right)$ above which the bundle fails completely. Recently it has been shown analytically [15, 16] that for uniform fiber threshold distribution, bundle's strength approaches the value $1 / 4$ as system size goes to infinity. On the other hand LLS bundles do not have any nonzero strength 21, 22, 23]. In our MMLS model we intend to study the strength variation of bundles with system size as well as with the weight parameter $g$.

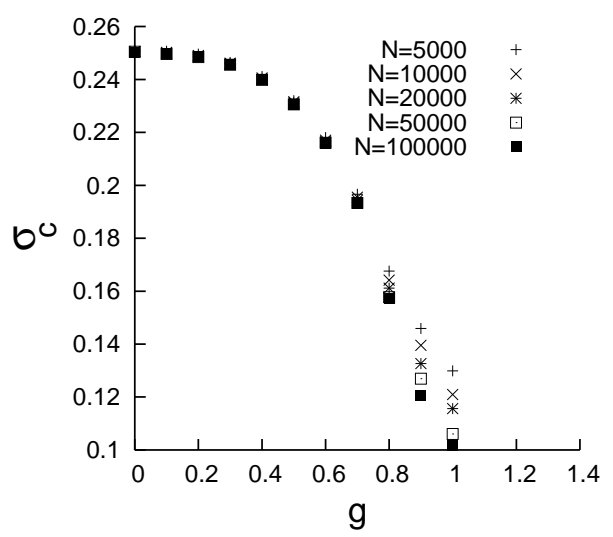

Fig. 1: The strength of the bundle for different system sizes $(N)$ as a function of the weight parameter $g$.

As $g$ increases, the bundle becomes weaker due to the short-range (LLS) interactions. Therefore $\sigma_{c}$ decreases with increasing $g$ values (Fig. 1). We can see that $\sigma_{c}$ seems to be independent on system size (dominance of ELS) up to $g=0.7$ and beyond $g=0.8$, a strong system size dependence (dominance of LLS) appears. This observation is supported by Fig. 2, where we have shown the logarithmic size dependence of $\sigma_{c}$. Up to $g=0.7$, the curves eventually become flat as the system size increases. But for $g \geq 0.8$, all the curves fall (following inclined straight line). Thus the 
two regimes are differentiated clearly.



Fig. 2: The logarithmic size dependence of bundle's strength for different values of $g$. The straight lines represent the best fit.

\section{B. Avalanche size distribution}

The avalanche size distribution characterizes the fracture process by reflecting the precursory activities toward complete failure. This can be related to the acoustic emissions observed in material failure [26, 27, 28]. Hemmer and Hansen showed [8] analytically that for ELS models the avalanche size distribution follows an universal power law with exponent value $-5 / 2$. But for LLS models the numerically estimated apparent exponent value is quite larger 4.5 9]. Later it has been shown analytically (for flat threshold distributions) that for LLS model, no universal power-law asymptotics exists [10].

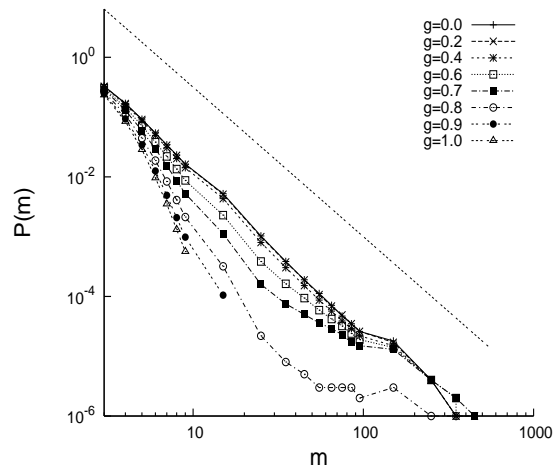

Fig. 3: Avalanche size distribution for different values of the weight parameter $g$ (averaging over 5000 configurations for system size $N=$
20000). The dotted line represents mean-field result having exponent value $-5 / 2$. Clearly, the upper group of curves can be fitted by the mean-field power law whereas the lower group does not show power law at all.

Here we have measured (Fig. 3) the avalanche size distributions for different $g$ values. Clearly, two groups of curves appear. The upper group $(0 \leq g \leq 0.7)$ can be fitted with the mean-field result $(-5 / 2)$ where as the lower group $(0.8 \leq g \leq 1.0)$ show a clear deviation from the power law.

\section{The susceptibility and relaxation time variations}

Recently, the dynamic response parameters, susceptibility $(\chi) \sqrt[13]{14}, 14,15,16,29]$ and relaxation time $(\tau)[15,16]$ have been studied in fiber bundle models. The susceptibility is defined as the number of fibers fail due to an infinitesimal change of the external stress $(\sigma)$ on the bundle and the relaxation time is the time (number of stress redistributions) the bundle takes to come to a stable fixed point at an external stress $(\sigma)$. For ELS model, the susceptibility and relaxation time seem to follow power law with the applied stress and both of them diverge 13, 15, 16, 17] at the critical strength $\sigma_{c}: \chi \sim\left(\sigma_{c}-\sigma\right)^{-1 / 2}$ and $\tau \sim\left(\sigma_{c}-\sigma\right)^{-1 / 2}$. However, one can not expect such scaling behavior in LLS models due to the absence of 'critical' strength. The step-wise equal load increment method [15, 16] enables to measure $\chi$ and $\tau$ for different values of $g$ (Fig. 4). The power law behavior (with mean-field exponent $-1 / 2$ ) remains unchanged up to $g=0.7$ and for $g \geq 0.8$ the curves do not follow power laws at all. Thus the susceptibility and relaxation time variations also suggest a transition from the mean-field to short range behavior to happen in between $g=0.7$ and $g=0.8$. 




Fig. 4: The susceptibility $(\chi)$ and relaxation time $(\tau)$ variations for different $g$ values. The bundle contains 10000 fibers and the data are averaged over 10000 configurations.

\section{Correlations among the broken fibers}

The breakdown sequence reflects the correlations of the breaking process 24]. While the ELS model simply ignores the spatial arrangement of the fibers, LLS model gives much importance on it. Therefore, as $g$ increases (LLS mode dominates) the breaking process becomes more and more correlated (Fig. 5). Here also we can identify two distinct regimes. We cannot see any spatial correlation among the broken fibers (except near the total failure) up to $g=0.7$, whereas for $g \geq 0.8$ strong correlations (black patch) develop long before the total failure.

$$
g=0.2
$$

$$
g=0.7
$$

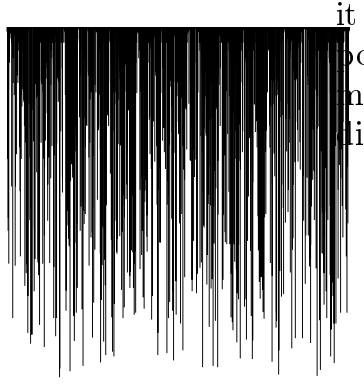

\section{DETERMINATION OF THE EXACT CROSSOVER POINT THROUGH CLUSTER MOMENT} ANALYSIS

The fracture process can also be characterized by analyzing the clusters of broken fibers just before complete failure [12, 25]. The size distributions of the clusters $(n(s)$ vs. $s)$ are shown (Fig. 6) for different values of $g$. Although the distributions appear as two groups, it is not possible to identify the exact crossover oint from this. Therefore we go for the monent analysis: the $k$-th moment of the cluster istributions is defined [25] as

$$
m_{k}=\int s^{k} n(s) d s
$$

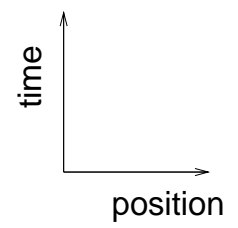

Clearly the zero-th moment $\left(m_{0}\right)$ gives the total number clusters and the first moment gives the total number of broken fibers. We can get the average cluster size dividing the second moment $\left(m_{2}\right)$ by first moment $\left(m_{1}\right)$. 


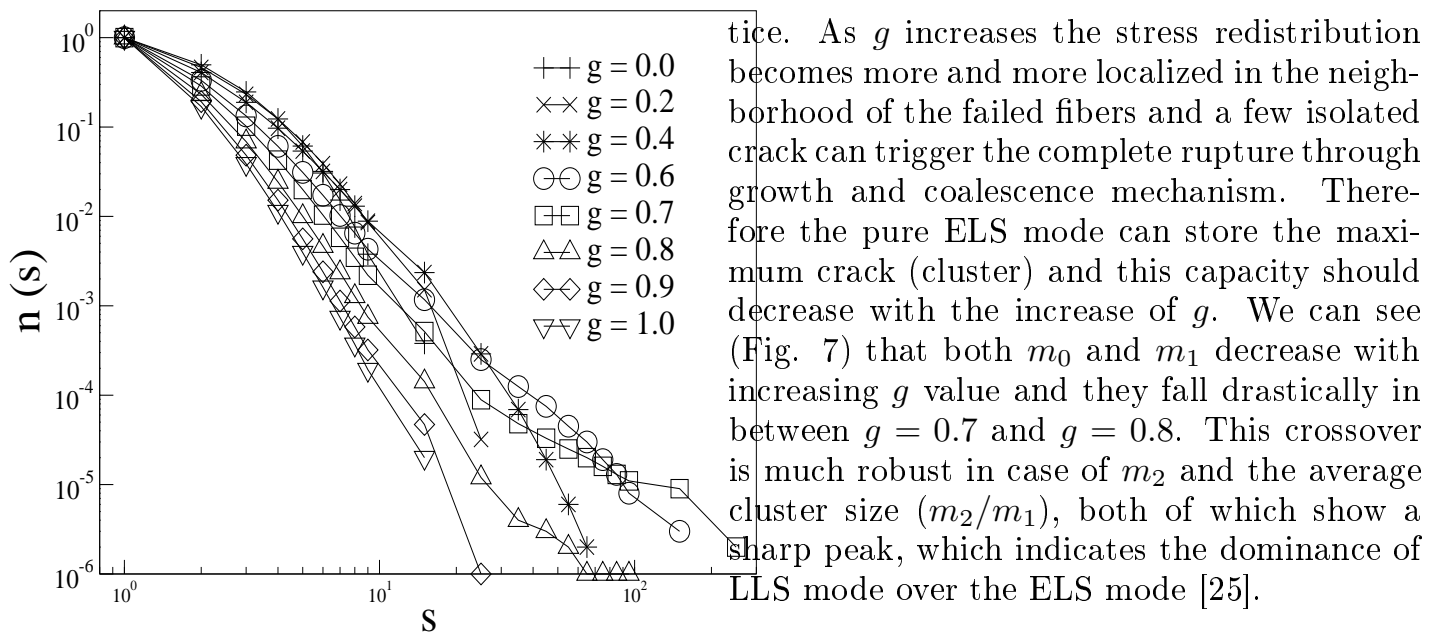

Fig. 6: Cluster size distributions of broken fibers (just before complete failure) for different $\mathrm{g}$ values (averaging over 5000 samples for $N=$ 20000).
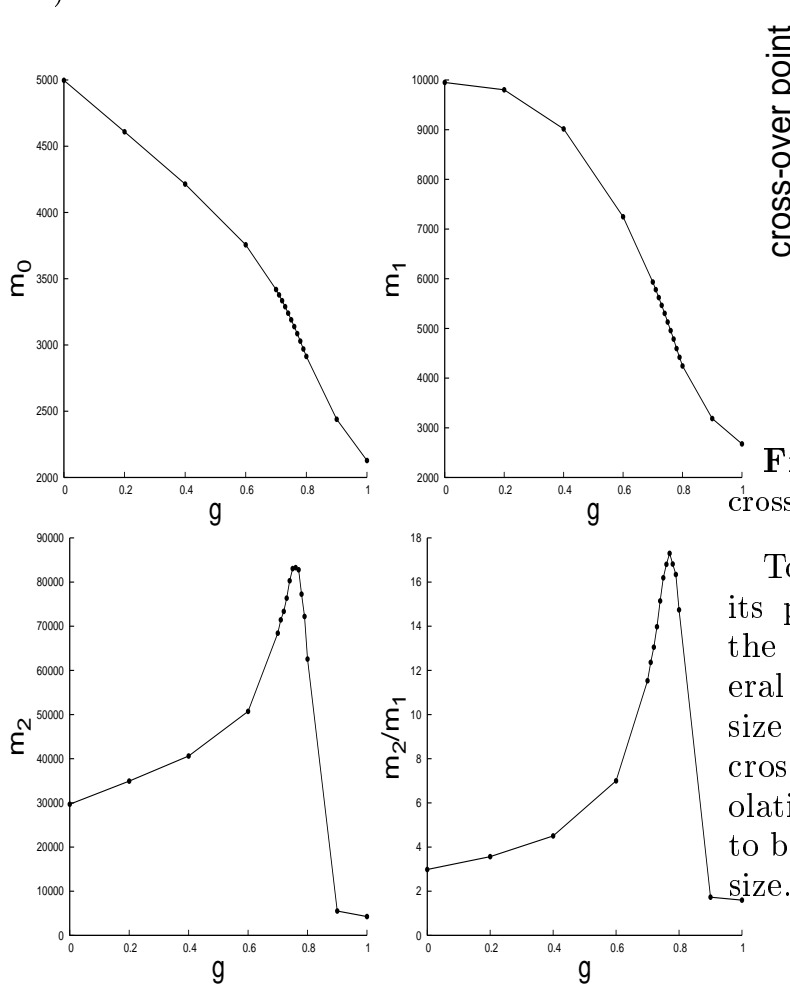

Fig. 7: The moments of the cluster size distributions as a function of the weight parameter $g$ (averaging over 5000 samples for $N=20000$ ) .

In case of pure ELS mode $(g=0)$, we have only long-range interaction and the clusters are randomly distributed within the lat-

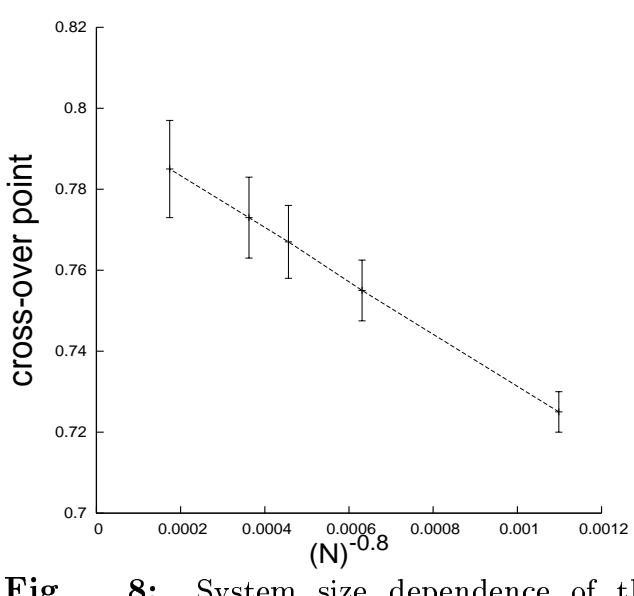

Fig. 8: System size dependence of the rossover point.

To check how the crossover point changes its position with system size, we have done the similar cluster moment analysis for several system sizes. We observe a weak system size dependence of the peak position i.e., the crossover point (Fig. 8). With proper extrapolation we determine the crossover point $\left(g_{c}\right)$ to be at $g=0.79 \pm 0.01$ for the infinite system sịize.

\section{CONCLUSION}

The fracture and breakdown of loaded materials is basically a cooperative phenomenon guided by the load redistribution mechanism. Here 'crack' opens up when an element (fiber) fails after external loading. This single fiber 
failure should affect the neighbors much than the distant elements (like in electric Fuse Models 1]). Therefore a high stress concentration (after the load redistribution) around a crack (failed fiber) is a natural expectation. The ELS models do not incorporate any spatial correlations and exhibit perfect democracy (mean-field), whereas the LLS models confine themselves within the nearest neighbor interactions. In this situation attempts [24, 25] to study the failure behavior in between ELS and LLS regimes, would be most welcome. Also, a recent experiment on loaded woodfiber [30] demands an intermediate load sharing scheme to explain the observed strength variation. The ' $\lambda$ model' $[24]$ becomes a LLS model at $\lambda=1$. But it cannot be reduced to a pure ELS model at $\lambda=0$, as the neighbors of the just broken fibers become 'immunized' against failure. Although the 'variable range of interaction' model [25] determines the exact crossover point, it remains silent about the system size dependence of crossover point, which is nevertheless an important issue.

Our mixed-mode load sharing (MMLS) model exactly reduces to ELS model at $g=0$ and to LLS model at $g=1$. We establish numerically that the MMLS model in one dimension shows a distinct crossover behavior from mean-field to short-range interaction. The strength $\left(\sigma_{c}\right)$ variation of the bundle with system size, the avalanche statistics and the failure dynamics (susceptibility and relaxation time) suggest that the crossover point $\left(g_{c}\right)$ must be in between $g=0.7$ and $g=0.8$. The cluster size analysis determines the exact crossover point in one dimension for several system sizes and a proper extrapolation suggests the crossover point to be $g_{c}=0.79 \pm 0.01$ at the limit of infinite system size. For $g<$ $g_{c}$ the model exhibits critical behavior (supported by the power laws) for the dominance of ELS mode. But the fluctuations suppress any critical behavior after $g=g_{c}$, where extreme statistics [1] dominates. We should mention that as the ultimate strength $\left(\sigma_{c}\right)$ of the bundle continuously decreases with the increasing $g$ value, we cannot exclude the possibility of different critical behavior for $g=0$, $0<g \leq g_{c}$ and $g>g_{c}$ in higher dimensions, like in case of $2-D$ Ising systems with disorder [31]. Therefore we expect this crossover behavior in MMLS model to be more prominent in higher dimensions.

Acknowledgment: We are grateful to Dr. M. Kloster for useful comments. S. P. thanks the Norwegian Research Council, NFR for the funding through a strategic university program.
[1] H. J. Herrmann and S. Roux (Eds), Statistical Models for the Fracture of Disordered Media, North Holland, Amsterdam (1990); B. K. Chakrabarti and L. G. Benguigui, Statistical Physics of Fracture and Breakdown in Disorder Systems, Oxford Univ. Press, Oxford (1997); M. Sahimi, Heterogeneous Materials II: Nonlinear and Breakdown Properties, Springer-Verlag, New York (2003).

[2] F. T. Peirce, J. Textile Inst. 17, T355-368 (1926).

[3] H. E. Daniels, Proc. R. Soc. London A 183 405 (1945).

[4] B. D. Coleman, J. Appl. Phys. 27, 862 (1956); B. D. Coleman, Trans. Soc. Rheol. 1, 153 (1957); B. D. Coleman, Trans. Soc. Rheol. 2, 195 (1958).

[5] W. I. Newman and S. L. Phoenix, Phys. Rev.
E 63, 021507 (2000).

[6] S. Roux, Phys. Rev. E 62, 6164 (2000); R. Scorretti, S. Ciliberto and A. Guarino, Europhys. Lett. 55, 626 (2001).

[7] S. Pradhan and B. K. Chakrabarti, Phys. Rev. E 67, 046124 (2003).

[8] P. C. Hemmer and A. Hansen, J. Appl. Mech. 59909 (1992).

[9] A. Hansen and P. C. Hemmer, Phys. Lett. A 184394 (1994).

[10] M. Kloster, A. Hansen and P. C. Hemmer, Phys. Rev. E 562615 (1997).

[11] D. Sornette, J. Phys. A 22 L243 (1989); D. Sornette, J. Phys. I (France) 22089 (1992); A. T. Bernardes and J. G. Moreira, Phys. Rev. B 4915035 (1994).

[12] S. Zapperi, P. Ray, H. E. Stanley and A. Vespignani, Phys. Rev. Lett. 781408 (1997); 
[13] Y. Moreno, J. B. Gomez and A. F. Pacheco, Phys. Rev. Lett. 852865 (2000).

[14] R. da Silveira, Am. J. Phys. 671177 (1999).

[15] S. Pradhan and B. K. Chakrabarti, Phys. Rev. E 65, 016113 (2001);

[16] S. Pradhan, P. Bhattacharyya and B. K. Chakrabarti, Phys. Rev. E 66, 016116 (2002);

[17] P. Bhattacharyya, S. Pradhan and B. K. Chakrabarti, Phys. Rev. E 67, 046122 (2003);

[18] D. G. Harlow and S. L. Phoenix, J. Composite Mater. 12, 314 (1978); R. L. Smith, Adv, Appl. Prob. 15, 304 (1982).

[19] S. L. Phoenix, Adv. Appl. Prob. 11, 153 (1979); R. L. Smith and S. L. Phoenix, J. Appl. Mech. 48, 75 (1981).

[20] S. L. Phoenix and R. L. Smith, J. Solid Struct. 19, 479 (1983).

[21] R. L. Smith, Proc. R. Soc. London A 372, 539 (1980).

[22] J. B. Gomez, D. Iniguez and A. F. Pacheco, Phys. Rev. Lett. 71, 380 (1993).

[23] S. Pradhan and B. K. Chakrabarti, Int. J.
Mod. Phys. B 175565 (2003).

[24] A. Hansen and P. C. Hemmer, Trends in Stat. Phys. 1, 213 (1994).

[25] R. C. Hidalgo, Y. Moreno, F. Kun and H. J. Herrmann, Phys. Rev. E 65046148 (2002).

[26] A. Garcimartin, A. Guarino, L. Bellon and S. Ciliberto, Phys. Rev. Lett. 793202 (1997).

[27] A. Guarino, A. Garcimartin and S. Ciliberto, Eur. Phys. J. B 613 (1998).

[28] A. Petri, G. Paparo, A. Vespignani, A. Alippi and M. Costantini, Phys. Rev. Lett 733423 (1994).

[29] M. Acharyya and B. K. Chakrabarti, Physica A 224254 (1996).

[30] G. D. Langer, R. C. Hidalgo, F. Kun, Y. moreno, S. Aicher and H. J. Herrmann, Physica A 325547 (2003).

[31] R. Stnchcombe, in C. Domb and J. L. Lebowitz, Phase Transition and Critical Phenomena, Vol. 7151 (1983). 